\title{
CATACLYSMIC VARIABLES FROM SLOAN DIGITAL SKY SURVEY. VI. THE SIXTH YEAR (2005) ${ }^{1}$

\author{
Paula Szkody, ${ }^{2}$ Arne Henden, ${ }^{3,4}$ Lee Mannikko, ${ }^{2}$ Anjum Mukadam, ${ }^{2}$ Gary D. Schmidt, ${ }^{5}$ John J. Bochanski, ${ }^{2}$ \\ Marcel Agüeros, ${ }^{2}$ Scott F. Anderson, ${ }^{2}$ Nicole M. Silvestri, ${ }^{2}$ William E. Dahab, ${ }^{6}$ Masamune Oguri, ${ }^{6,7}$ \\ Donald P. Schneider, ${ }^{8}$ Min-Su Shin, ${ }^{6}$ Michael A. Strauss, ${ }^{6}$ Gillian R. Knapp, ${ }^{6}$ and Andrew A. West ${ }^{2}$ \\ Received 2007 January 5; accepted 2007 March 28
}

\begin{abstract}
The 28 cataclysmic variables (CVs) found in 2005 in the Sloan Digital Sky Survey are presented with their coordinates, magnitudes, and spectra. Five of these systems are previously known CVs (HH Cnc, SX LMi, QZ Ser, $\mathrm{AP} \mathrm{CrB}$, and HS 1016+3412), and the rest are new discoveries. Additional spectroscopic, photometric, and/or polarimetric observations of 10 systems were carried out, resulting in estimates of the orbital periods for seven of the new binaries. The 23 new CVs include one eclipsing system, one new Polar, and five systems whose spectra clearly reveal atmospheric absorption lines from the underlying white dwarf.
\end{abstract}

Key words: binaries: eclipsing — binaries: spectroscopic — novae, cataclysmic variables — stars: dwarf novae

\section{INTRODUCTION}

The Sloan Digital Sky Survey (SDSS; York et al. 2000) has recently produced Data Release 5 , which includes photometry of 215 million sources over $8000 \mathrm{deg}^{2}$ and spectroscopy of over 1 million objects (Adelman-McCarthy et al. 2007). Previous releases are detailed by Stoughton et al. (2002), Abazajian et al. (2003, 2004, 2005), and Adelman-McCarthy et al. (2006). ${ }^{9}$ Among the many results stemming from the SDSS data is the discovery of a significant number of cataclysmic variables (CVs), the short-period binary stars in which a white dwarf accretes matter from a companion which usually resembles a late-type main-sequence star (all types of CVs are reviewed by Warner [1995], while those with magnetic white dwarfs are reviewed by Wickramasinghe \& Ferrario [2000]). The CV population has been found to consist primarily of short orbital period (under $2 \mathrm{hr}$ ) systems with very low mass transfer rates (Szkody et al. 2002, 2003a, 2003b, 2004, 2005, 2006; Schmidt et al. 2005, 2007). Some of these rates are so low that the accretion occurs by a wind from the secondary, rather than a stream of material from a filled Roche lobe. In addition, at these low rates, the accretion disk or column is so faint that the photosphere of the underlying stars can be seen spectroscopically.

This paper continues the series of yearly identifications of CVs in SDSS. We provide accurate coordinates, SDSS magnitudes and colors, and the SDSS spectra, along with some additional observations to improve the classifications. Since the list is large and continually growing, it will require an extensive

\footnotetext{
${ }^{1}$ Based on observations obtained with the Sloan Digital Sky Survey and the Apache Point Observatory $3.5 \mathrm{~m}$ telescope, which are owned and operated by the Astrophysical Research Consortium.

2 Department of Astronomy, University of Washington, Box 351580, Seattle, WA 98195, USA.

${ }^{3}$ US Naval Observatory, Flagstaff Station, P.O. Box 1149, Flagstaff, AZ 86002-1149, USA.

${ }^{4}$ American Association of Variable Star Observers, 49 Bay State Road, Cambridge, MA 02138, USA.

5 Steward Observatory, University of Arizona, Tucson, AZ 85721, USA.

${ }^{6}$ Princeton University Observatory, Peyton Hall, Princeton, NJ 08544, USA.

7 Kavli Institute for Particle Astrophysics and Cosmology, Stanford University, 2575 Sand Hill Road, Menlo Park, CA 94025, USA.

8 Department of Astronomy and Astrophysics, Pennsylvania State University, 525 Davey Laboratory, University Park, PA 16802, USA.

${ }^{9}$ Data are available from http://www.sdss.org.
}

observational campaign by many in the community to obtain good orbital periods and classifications for all sources. If this can be accomplished for all the objects, and the biases in the spectroscopic selection can be correctly determined, the SDSS data can ultimately provide a basis for rigorous tests of stellar evolution models for close binaries (Howell et al. 2001) that predict the numbers of systems over the range of orbital periods.

\section{OBSERVATIONS AND REDUCTIONS}

The details concerning the SDSS imaging and spectroscopic instrumentation and reductions are described by Szkody et al. (2002) and by several SDSS papers. As a brief summary, SDSS astrometric (Pier et al. 2003) and photometric (Gunn et al. 1998, 2006; Lupton et al. 1999, 2001; Hogg et al. 2001; Ivezic et al. 2004; Tucker et al. 2006) pipelines provide magnitudes in five filters ( $u, g, r, i$, and $z$; Fukugita et al. 1996; Smith et al. 2002). Objects are chosen for spectroscopic observations by a number of selection algorithms (see Stoughton et al. 2002). These algorithms cover color loci of quasars (Richards et al. 2002), cool stars, white dwarfs, serendipity, and white dwarf plus cool dwarf binaries. While this covers a wide range of color space, it does not guarantee that all CVs are targeted for spectra. The SDSS spectra cover $3800-9200 \AA$ at a resolving power of $\sim 1800$, and the pipelines provide wavelength and flux calibrations. The spectra are then classified (as, e.g., star, galaxy, or quasar) by an automatic software package.

CVs are identified in the SDSS spectra through an algorithm that selects objects with Balmer and helium emission/absorption lines at zero redshift, and the resulting objects are then classified by eye. As a cross-check, all spectra are viewed on some plates to see whether the algorithm misses any candidates. We estimate that we find more than $90 \%$ of the CVs that exist in the SDSS spectra, with a few missed through misidentification or low $\mathrm{S} / \mathrm{N}$ of the spectra. Table 1 lists the 23 new CVs, along with the 5 previously known ones, that had SDSS spectra obtained from 2005 January 1 through December 31, giving the plate, fiber, and Modified Julian Date (MJD) of each spectrum. The coordinates are equinox J2000.0 and use the IAU convention of truncation rather than rounding at the given decimal. The coordinates have an astrometric accuracy of $0.10^{\prime \prime}$. The magnitudes and colors are from the point-spread function photometry with no correction for interstellar reddening. To simplify the designations 
TABLE 1

CVS IN SDSS

\begin{tabular}{|c|c|c|c|c|c|c|c|c|}
\hline SDSSJ $^{\mathrm{a}}$ & MJD-P-F & $g$ & $u-g$ & $g-r$ & $r-i$ & $i-z$ & $\begin{array}{c}P \\
(\mathrm{hr})\end{array}$ & Comments $^{\mathrm{c}}$ \\
\hline $032855.00+052254.2$. & $53,713-2334-581$ & 18.03 & 0.49 & -0.06 & 0.01 & 0.36 & 2.03 & Polar \\
\hline 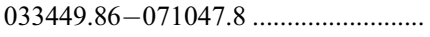 & $\ldots$ & 14.64 & 0.23 & -0.19 & -0.18 & -0.18 & 1.90 & $\mathrm{DN}$ \\
\hline $033710.91-065059.4 \ldots \ldots \ldots \ldots \ldots \ldots \ldots \ldots$ & $53,415-2070-320$ & 19.57 & 0.12 & -0.19 & -0.23 & -0.18 & $\ldots$ & \\
\hline 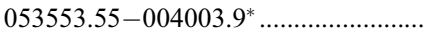 & $53,430-2072-060$ & 19.42 & 0.07 & -0.13 & -0.23 & -0.25 & $\ldots$ & \\
\hline 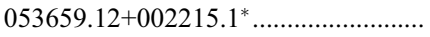 & $53,401-2052-593$ & 18.82 & 0.46 & -0.15 & -0.16 & -0.23 & $\ldots$ & \\
\hline $061542.53+642647.6 \ldots \ldots \ldots$ & $53,712-2301-544$ & 19.64 & 1.52 & 0.67 & 0.33 & 0.21 & $\ldots$ & \\
\hline $075059.97+141150.1 \ldots \ldots \ldots .$. & $53,682-2264-352$ & 19.08 & 0.11 & 0.10 & 0.18 & 0.21 & $\ldots$ & \\
\hline $075507.70+143547.6 \ldots \ldots \ldots \ldots \ldots$ & $53,682-2264-416$ & 18.23 & -0.09 & -0.07 & -0.15 & 0.02 & 1.4 & \\
\hline $080534.49+072029.1 * \ldots \ldots \ldots \ldots \ldots \ldots \ldots \ldots$ & $53,442-2076-501$ & 18.52 & 1.55 & 0.73 & 0.26 & 0.15 & $\ldots$ & \\
\hline $081207.63+131824.4$. & $53,682-2268-069$ & 19.25 & -0.03 & 0.11 & 0.10 & 0.38 & $\ldots$ & \\
\hline $083754.64+564506.7^{*} \ldots \ldots$ & $53,386-1783-623$ & 18.97 & 0.61 & -0.01 & -0.09 & -0.06 & $\ldots$ & \\
\hline 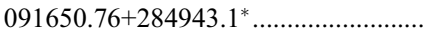 & $53,388-1937-416$ & 19.17 & -0.19 & 0.65 & 0.34 & 0.27 & $\ldots$ & $\mathrm{HH} \mathrm{Cnc}$ \\
\hline $094002.56+274942.0^{*} \ldots \ldots \ldots \ldots \ldots \ldots \ldots \ldots$ & $53,325-1944-190$ & 19.10 & 0.55 & 0.77 & 0.40 & 0.30 & $\cdots$ & \\
\hline $094558.24+292253.2^{*} \ldots \ldots$. & $53,431-1947-400$ & 19.10 & 0.11 & -0.08 & -0.37 & -0.01 & $\ldots$ & \\
\hline 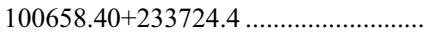 & $53,735-2343-493$ & 18.34 & 0.13 & 0.40 & 0.42 & 0.38 & $\ldots$ & \\
\hline 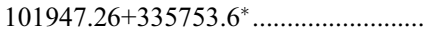 & $53,442-1955-529$ & 18.39 & -0.55 & 0.19 & 0.14 & 0.15 & 1.91 & HS $1016+3412$ \\
\hline 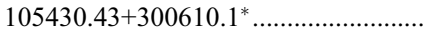 & $53,463-1981-066$ & 16.76 & -0.49 & 0.25 & 0.12 & 0.04 & 1.61 & SX LMi \\
\hline 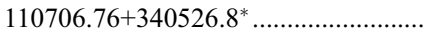 & $53,466-2034-215$ & 19.48 & 0.18 & 0.63 & 0.22 & 0.46 & $\ldots$ & \\
\hline 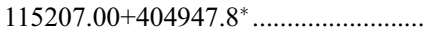 & $53,386-1970-541$ & 19.27 & -0.09 & 0.12 & 0.01 & 0.15 & $\ldots$ & \\
\hline 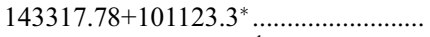 & $53,533-1709-153$ & 18.55 & -0.04 & 0.12 & -0.10 & 0.03 & 1.30 & Ec \\
\hline 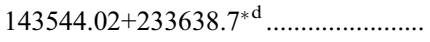 & $53,494-2136-541$ & 18.24 & 0.12 & -0.03 & -0.22 & -0.14 & 1.3 & \\
\hline $152717.96+543724.9^{*} \ldots \ldots \ldots \ldots \ldots \ldots \ldots \ldots \ldots \ldots \ldots \ldots \ldots \ldots$ & $53,437-0614-251$ & 20.39 & -0.23 & 0.05 & -0.08 & -0.04 & $\ldots$ & \\
\hline 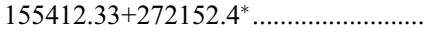 & $53,498-1654-542$ & 17.60 & 0.05 & -0.14 & -0.25 & 0.75 & 2.53 & AP CrB Polar \\
\hline 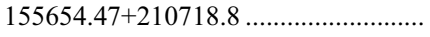 & $53,557-2171-007$ & 17.92 & 0.56 & 0.75 & 0.32 & 0.23 & 2.00 & QZ Ser \\
\hline 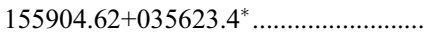 & $53,494-1837-283$ & 18.41 & -0.17 & -0.17 & -0.09 & -0.01 & $\cdots$ & \\
\hline 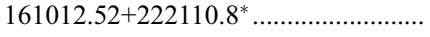 & $53,534-1852-153$ & 17.43 & 0.37 & -0.03 & -0.12 & -0.06 & $\ldots$ & KUV $16081+2229$ \\
\hline $164248.52+134751.4^{*} \ldots \ldots \ldots \ldots \ldots \ldots \ldots \ldots \ldots \ldots \ldots \ldots \ldots$ & $53,535-2210-592$ & 18.64 & -0.16 & 0.15 & 0.08 & 0.21 & 1.3 & \\
\hline 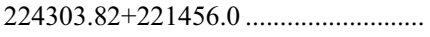 & $53,565-2252-239$ & 17.03 & 0.10 & -0.17 & -0.16 & -0.23 & $\ldots$ & He II \\
\hline
\end{tabular}

a Objects marked with an asterisk are publicly available in the SDSS DR5.

b MJD-Plate-Fiber for spectra; MJD $=$ JD $-2,450,000.5$.

c $\mathrm{DN}$ is a dwarf nova, and Ec is eclipsing.

d Object is the eastern star of a close pair.

throughout this paper, we refer to the objects as SDSSJ hhmm (hours and minutes of right ascension). One object in Table 1 (SDSSJ 0334) has no SDSS spectrum. It was discovered serendipitously in a search for bright $(i<15) \mathrm{UV}$-excess quasars in the SDSS. The SDSS images of this object are saturated or nearly so in several bands, but the SDSS photometry remains good for a few magnitudes beyond saturation, as described in Abazajian et al. (2004).

The available follow-up observations of these systems are listed in Table 2. Differential photometry was obtained at the Sonoita Research Observatory (SRO) with the $0.35 \mathrm{~m}$ telescope, using an SBIG STL-1001E camera with a $1024 \times 1024$ CCD. Additional photometry on SDSSJ 0334 was obtained at the US Naval Observatory Flagstaff Station (NOFS) with the $1.0 \mathrm{~m}$ telescope and $1024 \times 1024 \mathrm{SITe} /$ Tektronix CCD. No filter was used for the SRO or NOFS observations; these CCDs have approximately wide-band Johnson $V$ magnitude response when used unfiltered. The fields were calibrated with other nights of all-sky photometry using Landolt standards so that the comparison stars could be placed onto the Johnson $V$ magnitude system. The data on SDSSJ 0334 are calibrated from a photometric night, whereas SDSSJ 0328 and 2243 have less accurate zero points determined.

Two nights of photometry on the Apache Point Observatory (APO) $3.5 \mathrm{~m}$ telescope using the Dual Imaging Spectrograph (DIS) in imaging mode with no filters were obtained for SDSSJ 1443. This object was calibrated against $g$-magnitudes for other SDSS stars in the field.

Several other objects were observed for brief intervals on single nights, using the DIS in spectroscopic mode. Most of these
TABLE 2

Follow-UP DATA

\begin{tabular}{|c|c|c|c|c|c|}
\hline SDSSJ & UT Date & Site & $\begin{array}{l}\text { Time } \\
\text { (UT) }\end{array}$ & $\begin{array}{c}\text { Exp. } \\
\text { (s) }\end{array}$ & Data Obtained \\
\hline $0328 \ldots \ldots$ & 2005 Dec 30 & Bok & $05: 27-07: 22$ & 6600 & Circular polarimetry \\
\hline $0328 \ldots \ldots$ & 2006 Feb 1 & SRO & $02: 24-06: 01$ & 240 & Photometry \\
\hline $0328 \ldots \ldots$ & 2006 Feb 2 & SRO & $02: 10-06: 14$ & 240 & Photometry \\
\hline $0334 \ldots \ldots$ & 2005 Dec 27 & $\mathrm{APO}$ & 05:04 & 300 & 1 spectrum \\
\hline $0334 \ldots \ldots$ & 2006 Jan 27 & NOFS & $02: 03-06: 29$ & 80 & Photometry \\
\hline $0334 \ldots \ldots$ & 2006 Feb 3 & SRO & $02: 12-05: 23$ & 240 & Photometry \\
\hline $0334 \ldots \ldots$ & 2006 Feb 4 & SRO & $02: 17-05: 27$ & 240 & Photometry \\
\hline $0334 \ldots \ldots$ & 2006 Oct 22 & APO & $06: 15-08: 48$ & 600 & 13 spectra \\
\hline $0750 \ldots \ldots$ & 2005 Dec 8 & APO & $11: 12-12: 35$ & 600 & 3 spectra \\
\hline $0755 \ldots \ldots$ & 2005 Dec 8 & $\mathrm{APO}$ & $08: 20-10: 56$ & 600 & 13 spectra \\
\hline $1019 \ldots \ldots$ & 2006 May 29 & $\mathrm{APO}$ & $03: 06-04: 54$ & 600 & 9 spectra \\
\hline $1107 \ldots .$. & 2006 May 29 & APO & $04: 58-06: 38$ & 600 & 9 spectra \\
\hline $1433 \ldots \ldots$ & 2006 May 3 & APO & 07:11-10:03 & 10 & DIS imaging \\
\hline $1433 \ldots \ldots$ & 2006 May 30 & $\mathrm{APO}$ & 03:07-07:42 & 15 & DIS imaging \\
\hline $1435 \ldots$. & 2006 May 23 & APO & $07: 38-09: 26$ & 600 & 9 spectra \\
\hline $1642 \ldots \ldots$ & 2006 May 23 & $\mathrm{APO}$ & $09: 45-11: 05$ & 600 & 7 spectra \\
\hline $2243 \ldots \ldots$ & 2006 Oct 22 & $\mathrm{APO}$ & $01: 47-03: 40$ & 600 & 10 spectra \\
\hline $2243 \ldots \ldots$ & 2006 Nov 23 & SRO & $03: 15-05: 45$ & 240 & Photometry \\
\hline $2243 \ldots \ldots$ & 2006 Nov 25 & SRO & 02:42-06:08 & 240 & Photometry \\
\hline
\end{tabular}


TABLE 3

SDSS Emission-Line FluXes and Equivalent Widths

\begin{tabular}{|c|c|c|c|c|c|c|c|c|}
\hline \multirow[b]{2}{*}{ SDSSJ } & \multicolumn{2}{|c|}{$\mathrm{H} \beta$} & \multicolumn{2}{|c|}{$\mathrm{H} \alpha$} & \multicolumn{2}{|c|}{ He $\lambda 4471$} & \multicolumn{2}{|c|}{ He II $\lambda 468$} \\
\hline & $F$ & EW & $F$ & EW & $F$ & EW & $F$ & EW \\
\hline $328 \ldots$ & 3.5 & 21 & 2.6 & 26 & & 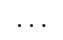 & 3.4 & 20 \\
\hline $334 \ldots$ & 8.7 & 44 & 16.4 & 76 & 1.3 & 8 & $\ldots$ & $\ldots$ \\
\hline $0337 \ldots$ & 0.3 & 73 & 0.2 & 330 & $\ldots$ & $\ldots$ & $\ldots$ & $\ldots$ \\
\hline $0535 \ldots$ & 0.1 & 2 & 0.4 & 17 & $\ldots$ & $\ldots$ & $\ldots$ & \\
\hline $0536 \ldots \ldots$. & $\ldots$ & $\ldots$ & 0.3 & 9 & $\ldots$ & $\ldots$ & $\ldots$ & \\
\hline $0615 \ldots \ldots \ldots \ldots \ldots \ldots$ & 0.1 & 3 & 0.6 & 12 & $\ldots$ & $\ldots$ & $\ldots$ & \\
\hline $0750 \ldots \ldots$ & 3.6 & 35 & 4.5 & 67 & $\ldots$ & $\ldots$ & $\ldots$ & \\
\hline $755 \ldots \ldots \ldots \ldots \ldots \ldots$ & 3.8 & 31 & 8.4 & 122 & $\ldots$ & $\ldots$ & $\ldots$ & \\
\hline $0805 \ldots \ldots \ldots \ldots \ldots \ldots$ & 1.0 & 7 & 1.9 & 13 & $\ldots$ & $\ldots$ & $\ldots$ & $\ldots$ \\
\hline $0812 \ldots$ & 6.0 & 44 & 6.7 & 72 & 1.3 & 8 & 0.7 & 6 \\
\hline 0837..................... & 0.4 & 38 & 0.4 & 52 & $\ldots$ & $\ldots$ & $\ldots$ & $\cdots$ \\
\hline 0916...................... & 2.9 & 60 & 3.8 & 55 & 0.7 & 13 & 0.2 & 3 \\
\hline $0940 \ldots$ & 4.2 & 35 & 6.5 & 53 & 1.2 & 10 & 1.1 & 10 \\
\hline $0945 \ldots$ & 3.6 & 41 & 4.0 & 105 & 0.9 & 8 & 0.2 & 2 \\
\hline $1006 \ldots$ & 3.7 & 38 & 6.2 & 62 & $\ldots$ & $\ldots$ & $\ldots$ & $\cdots$ \\
\hline $1019 \ldots$ & 10.7 & 93 & 11.7 & 137 & 2.3 & 19 & 1.1 & 11 \\
\hline $1054 \ldots \ldots$ & 32.4 & 88 & 30.2 & 116 & 6.7 & 17 & 3.1 & 8 \\
\hline $1107 \ldots \ldots \ldots \ldots \ldots \ldots$ & 6.4 & 45 & 10.4 & 85 & 1.3 & 9 & $\ldots$ & \\
\hline $1152 \ldots \ldots \ldots \ldots \ldots$ & 1.6 & 32 & 2.8 & 97 & $\ldots$ & $\ldots$ & $\ldots$ & \\
\hline $1433 \ldots \ldots$ & 5.1 & 51 & 9.6 & 154 & $\ldots$ & $\ldots$ & $\ldots$ & \\
\hline $1435 \ldots \ldots \ldots \ldots \ldots \ldots$ & 2.9 & 19 & 6.2 & 76 & $\ldots$ & $\ldots$ & $\ldots$ & \\
\hline $1527 \ldots \ldots \ldots \ldots \ldots$ & 0.7 & 31 & 1.2 & 100 & $\ldots$ & $\ldots$ & $\ldots$ & $\ldots$ \\
\hline $1554 \ldots \ldots \ldots \ldots \ldots$ & 14.7 & 29 & 20.0 & 68 & 2.7 & 7 & 4.2 & 10 \\
\hline $1556 \ldots \ldots \ldots \ldots \ldots \ldots$ & 4.0 & 27 & 6.0 & 38 & 1.4 & 9 & $\ldots$ & \\
\hline $1559 \ldots \ldots \ldots \ldots \ldots$ & 0.3 & 3 & 0.5 & 8 & $\ldots$ & $\ldots$ & $\ldots$ & \\
\hline $1610 \ldots$ & $\ldots$ & $\ldots$ & 0.4 & 3 & $\ldots$ & $\ldots$ & $\ldots$ & \\
\hline $1642 \ldots$ & 10.4 & 58 & 8.9 & 67 & 2.1 & 11 & 0.8 & 4 \\
\hline $2243 \ldots \ldots \ldots \ldots \ldots$ & 0.9 & 2 & 0.7 & 3 & $\ldots$ & $\ldots$ & 0.8 & 2 \\
\hline
\end{tabular}

Note.-Fluxes are in units of $10^{-15} \mathrm{ergs} \mathrm{cm}^{-2} \mathrm{~s}^{-1}$, and equivalent widths are in units of $\AA$.

spectra were obtained with high-resolution gratings (resolution about $2 \AA$ ) with a $1.5^{\prime \prime}$ slit over several hours and were used to construct radial velocity curves. One spectrum (2005 December 27 on SDSSJ 0334) was obtained with the medium-resolution gratings $(\sim 5 \AA)$ for classification purposes. All spectra were flux- and wavelength-calibrated using standard IRAF ${ }^{10}$ routines. The SDSS spectra were measured with the centroid-finding "e" routine in the IRAF splot package to obtain the equivalent widths and fluxes for the Balmer and helium emission lines (Table 3). For simple lines, this routine provided the centroid wavelengths of the lines for the APO data. For lines with good S/N but complicated structure (absorption or narrow emission structure within the line), the velocities were determined with the double-Gaussian method (Shafter 1983). If a plot of velocity versus time showed a sinusoidal variation during the $2-3 \mathrm{hr}$ of data, a least-squares fit to the velocities was used to find $\gamma$ (systemic velocity), $K$ (semiamplitude), $P$ (orbital period), and $T_{0}$ (the epoch of red to blue crossing of the systemic velocity), with the results listed in Table 4. Note that due to the short length of the data, most periods are only estimates and will need several nights of data for accurate determinations. However, they should provide a starting point as to whether the systems have short or long periods.

Circular polarization was measured for SDSSJ 0328, using the CCD spectropolarimeter SPOL with a low-resolution grating on the $2.3 \mathrm{~m}$ Bok telescope on Kitt Peak.

\footnotetext{
${ }^{10}$ IRAF (Image Reduction and Analysis Facility) is distributed by the National Optical Astronomy Observatory, which is operated by AURA, Inc., under cooperative agreement with the National Science Foundation.
}

TABLE 4

Radial Velocity Solutions

\begin{tabular}{|c|c|c|c|c|c|c|}
\hline SDSSJ & Line & $\begin{array}{c}P^{\mathrm{a}} \\
\text { (minutes) }\end{array}$ & $\gamma$ & $\begin{array}{c}K \\
\left(\mathrm{~km} \mathrm{~s}^{-1}\right)\end{array}$ & $\begin{array}{c}T_{0} \\
\text { (JD 2,450,000+) }\end{array}$ & \\
\hline $0328 .$. & $\mathrm{H} \alpha$ & $120 \pm 9$ & $44 \pm 19$ & $321 \pm 30$ & 3735.768 & 22 \\
\hline $0334 \ldots$ & $\mathrm{H} \alpha$ & 114 & $31 \pm 1$ & $46 \pm 5$ & 4030.800 & 12 \\
\hline $0334 \ldots \ldots \ldots$ & $\mathrm{H} \beta$ & 114 & $52 \pm 2$ & $73 \pm 6$ & 4030.803 & 15 \\
\hline $0755 \ldots \ldots \ldots$ & $\mathrm{H} \alpha$ & 83 & $-1.9 \pm 0.3$ & $62 \pm 5$ & 3712.867 & 12 \\
\hline $0755 \ldots \ldots \ldots$ & $\mathrm{H} \beta$ & 87 & $72 \pm 2$ & $97 \pm 14$ & 3712.869 & 35 \\
\hline $1019 \ldots \ldots \ldots$ & $\mathrm{H} \alpha$ & 104 & $62.5 \pm 0.1$ & $59 \pm 3$ & 3884.683 & 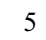 \\
\hline $1019 \ldots \ldots \ldots$ & $\mathrm{H} \beta$ & 117 & $58 \pm 1$ & $51 \pm 8$ & 3884.693 & 14 \\
\hline $1019 \ldots \ldots \ldots$ & $\mathrm{H} \alpha$ & 114.3 & $57.1 \pm 0.2$ & $62 \pm 3$ & 3884.685 & \\
\hline $1019 \ldots \ldots \ldots$ & $\mathrm{H} \beta$ & 114.3 & $60 \pm 1$ & $50 \pm 8$ & 3884.692 & 14 \\
\hline $1435 \ldots \ldots \ldots$ & $\mathrm{H} \alpha$ & 83 & $-14 \pm 3$ & $86 \pm 15$ & 3878.821 & 28 \\
\hline $1435 \ldots \ldots \ldots$ & $\mathrm{H} \beta$ & 74 & $34 \pm 5$ & $197 \pm 22$ & 3878.830 & 43 \\
\hline $1642 \ldots \ldots \ldots$ & $\mathrm{H} \alpha$ & 64 & $-84 \pm 2$ & $141 \pm 12$ & 3878.930 & 19 \\
\hline $1642 \ldots \ldots \ldots$ & $\mathrm{H} \beta$ & 77 & $-102 \pm 2$ & $145 \pm 32$ & 3878.931 & 52 \\
\hline
\end{tabular}

${ }^{a}$ Periods with whole integers are generally uncertain by $10 \%$, as evidenced by the dispersion between values obtained from the two lines.

\section{RESULTS}

The 27 systems with SDSS spectra are shown in Figure 1, and the equivalent widths and fluxes of the prominent hydrogen Balmer and helium emission lines are given for all 28 in Table 3. Using categories similar to those of the previous papers, we sort and present the systems with identifiable/interesting characteristics.

\subsection{Previously Known Systems}

The objects listed in Table 1 contain five known CVs: the four dwarf novae HH Cnc (SDSSJ 0916), SX LMi (SDSSJ 1054), QZ Ser (SDSSJ 1556), and HS 1016+3412 (SDSSJ 1019) and the highly magnetic CV (Polar) AP CrB (SDSSJ 1554, HS 1552+ 2730, RX J1554.2+2721). The general characteristics and literature references of $\mathrm{HH}$ Cnc, SX LMi, and QZ Ser are available from the online CV catalog of R. Downes. ${ }^{11} \mathrm{HH}$ Cnc has not been studied sufficiently to identify an orbital period, while the three other dwarf novae have periods below $2 \mathrm{hr}$ (Table 1). HH Cnc and SX LMi appear to be typical short-period dwarf novae with outbursts and superoutbursts (Kato \& Uemura 2001; Wagner et al. 1998), while QZ Ser is one of the rare peculiar short-period dwarf novae with a K-type secondary (Thorstensen et al. 2002).

AP CrB was originally found in the Hamburg Quasar Survey (Jiang et al. 2000). Tovmassian et al. (2001) identified it as a Polar with a period in the middle of the $\mathrm{CV}$ period gap between 2 and $3 \mathrm{hr}$ (the period was later refined to 151.865 minutes by Thorstensen \& Fenton 2002). Gänsicke et al. (2004) identified Zeeman split Ly $\alpha$ absorption from an HST STIS spectrum, revealing a high field strength of $144 \mathrm{MG}$. A recent paper (Schwope et al. 2006) has already discussed the SDSS spectrum and the cyclotron harmonic that is visible near $4800 \AA$.

HS $1016+3412$ was recently identified as a dwarf nova by Aungwerojwit et al. (2006, hereafter A06). They found one outburst (magnitude of 15.4) on 2004 November 2, while most of their photometry showed the system at $V \sim 17.5$, with one measurement at $V \sim 18.6$. The SDSS photometry shows a $g$-magnitude near their faintest measurement, while the SDSS spectrum looks similar to the one shown by A06. Our APO data were obtained prior to the publication of A06 and so were analyzed independently for a period. Figure 2 and Table 4 present our radial velocity results for the $\mathrm{H} \alpha$ and $\mathrm{H} \beta$ lines. A plot of the velocities versus time showed a clear sinusoidal motion with a period slightly

\footnotetext{
${ }^{11}$ See http://archive.stsci.edu/prepds/cvcat/index.html.
} 

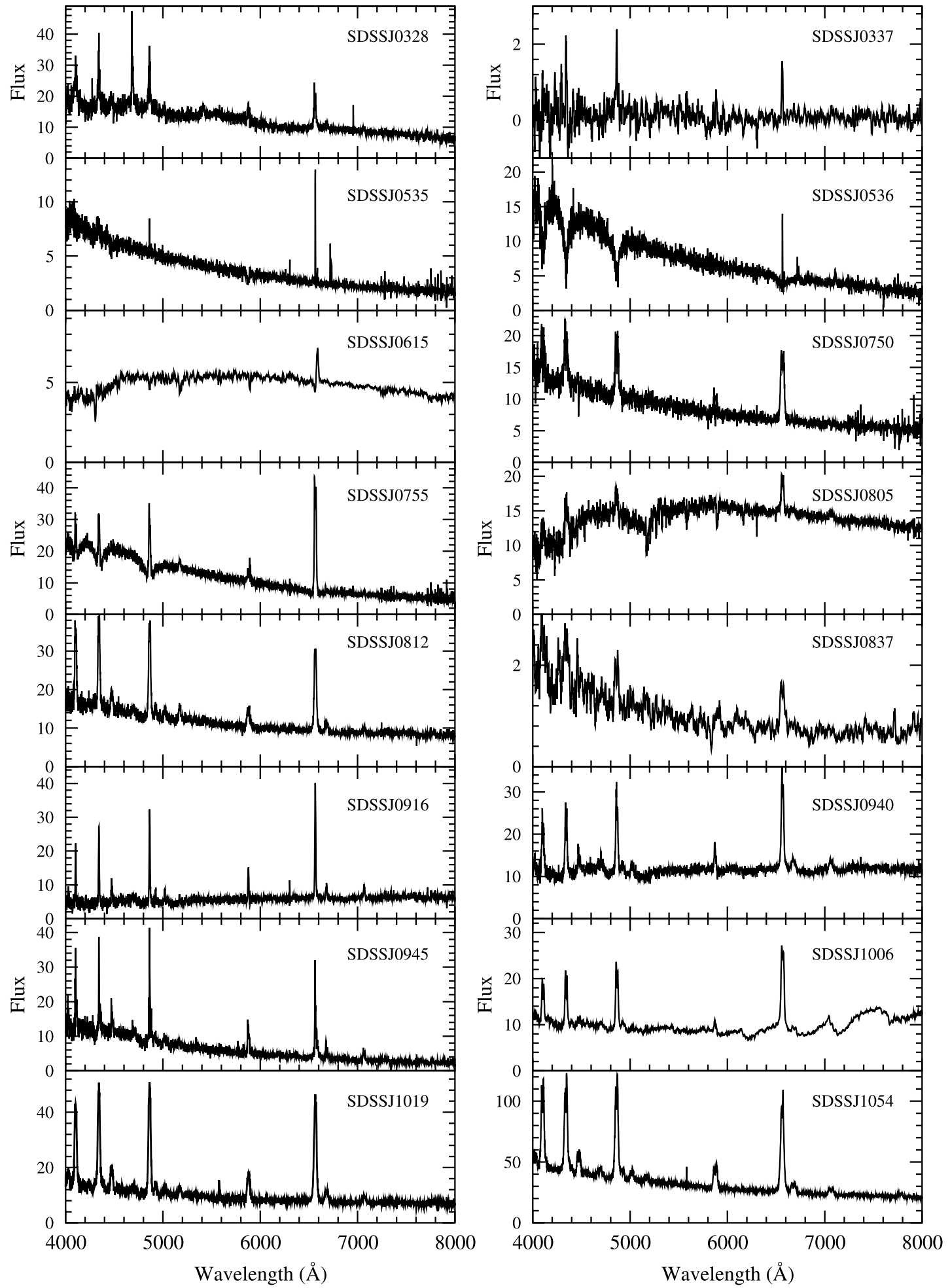

FIg. 1.-SDSS spectra of the $27 \mathrm{CVs}$. Vertical axis is units of flux density $F_{\lambda} \times 10^{-17} \mathrm{ergs} \mathrm{cm}^{-2} \mathrm{~s}^{-1} \AA^{-1}$. The spectral resolution is about $3 \AA$.

longer than our data set of 107 minutes. The results of A06 using a longer data set for $\mathrm{H} \alpha$ produced a period of $114.3 \pm 2.7$ minutes, a value that is consistent with our periods (within our estimated $10 \%$ error bars). Table 4 also shows our best sine wave fit with the period fixed at the 114.3 minute value. Our $K$ amplitude is less than that found by A06, but this value is a function of how the velocities are measured. A06 used a double Gaussian with a separation of $100 \mathrm{~km} \mathrm{~s}^{-1}$, whereas we used the centroid " $\mathrm{e}$ " routine in IRAF for our fit.

\subsection{High-Inclination Systems}

The systems with high inclination typically show an obvious double-peaked structure in their Balmer emission lines, with the central depression sometimes reaching to the continuum in the 

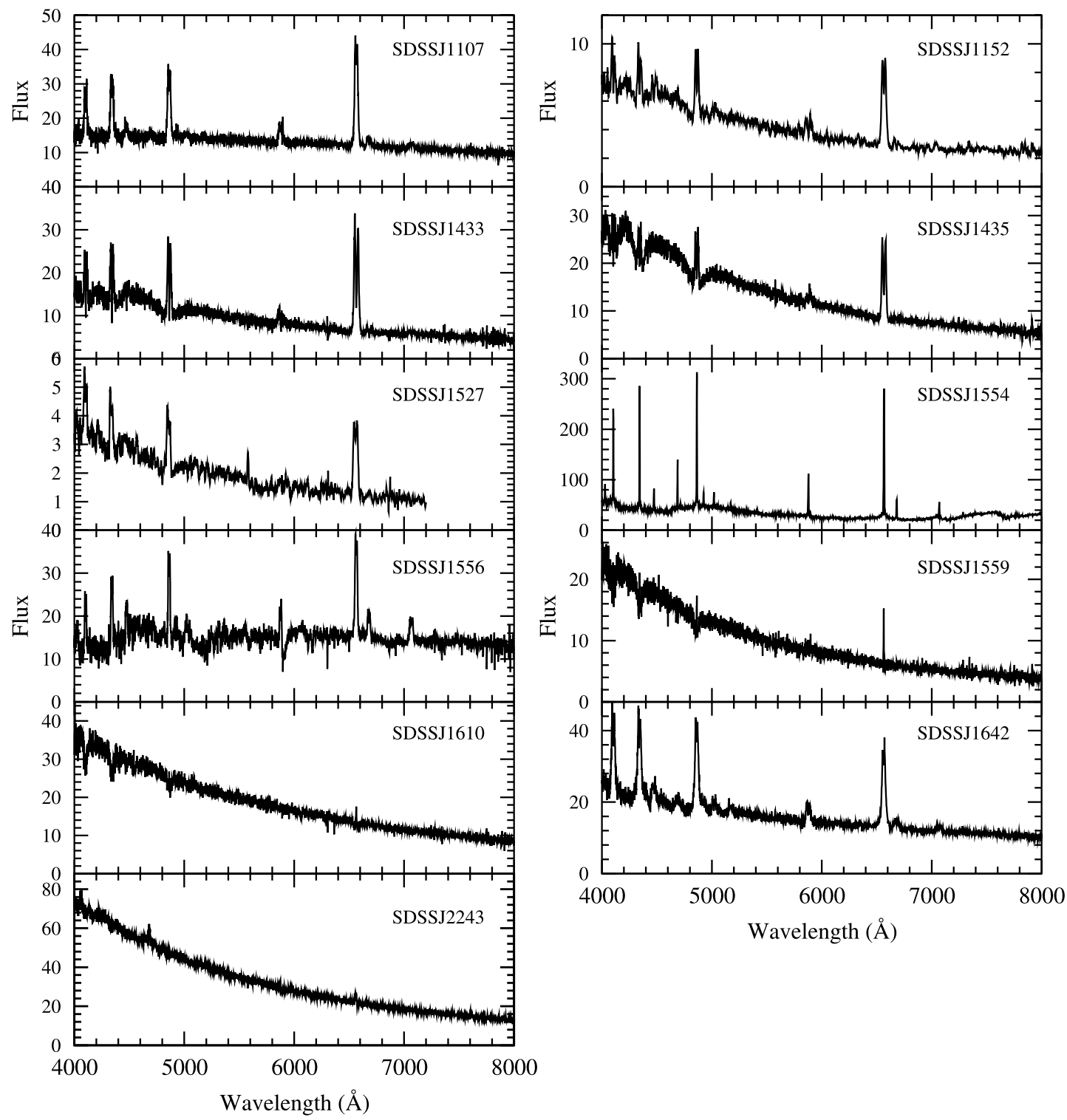

Fig. 1-Continued

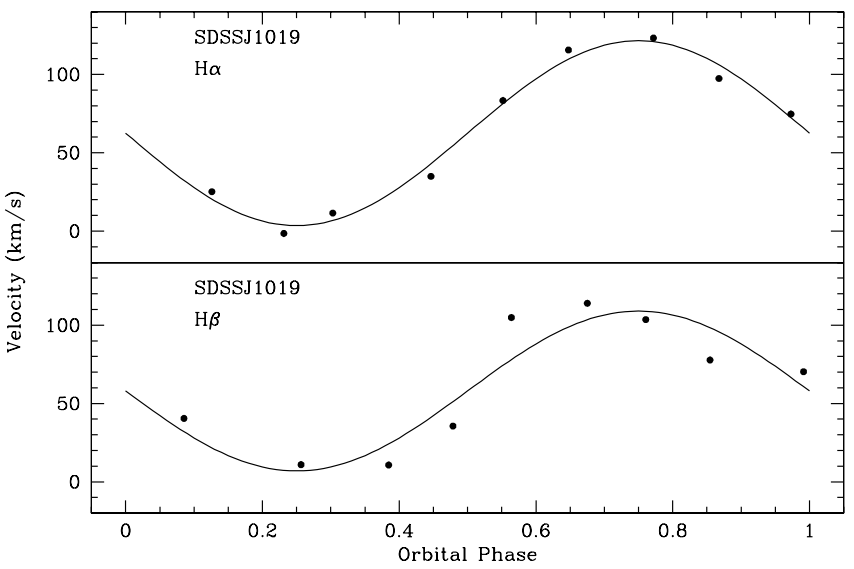

Fig. 2.- $\mathrm{H} \alpha$ and $\mathrm{H} \beta$ velocity curves of SDSSJ 1019 with the best-fit sinusoids (Table 4) superposed. Errors of fits are listed in Table 4. higher order lines. These features are readily apparent in SDSSJ 1152, 1433, and 1435 and to a lesser extent in SDSSJ 0750. Of these, only SDSSJ 1433 has follow-up photometric observations, which revealed eclipses (described below). APO spectra were able to find a period for SDSSJ 1435, but the sparse and low-S/N spectra of SDSSJ 0750 (Table 2) were not sufficient to determine the orbital period or whether eclipses are present.

SDSSJ 1433. - The two nights of APO photometry revealed six eclipses. The four eclipses on May 30 yield an orbital period of $78.12 \pm 0.45$ minutes (unfortunately, the two nights were too far separated to be used to refine the period further). Figure 3 shows the light curves on the two nights (May 3 and 30), and Table 5 lists all the eclipse times in TCB (these times are the faintest points of the light curve). The eclipse is deep (2.5 mag) and centered on a broad hump, indicating that there is some structure in the disk. The hump is not as strong as the typical hot-spot hump that results from the mass transfer stream impacting the disk (e.g., SDSSJ 1556; Szkody et al. 2006), nor is the light curve similar to the ultrashort-period CVs that show a double-humped light curve (e.g., SDSSJ 0804; Szkody et al. 2006) thought to be due to the impact stream and its overflow 


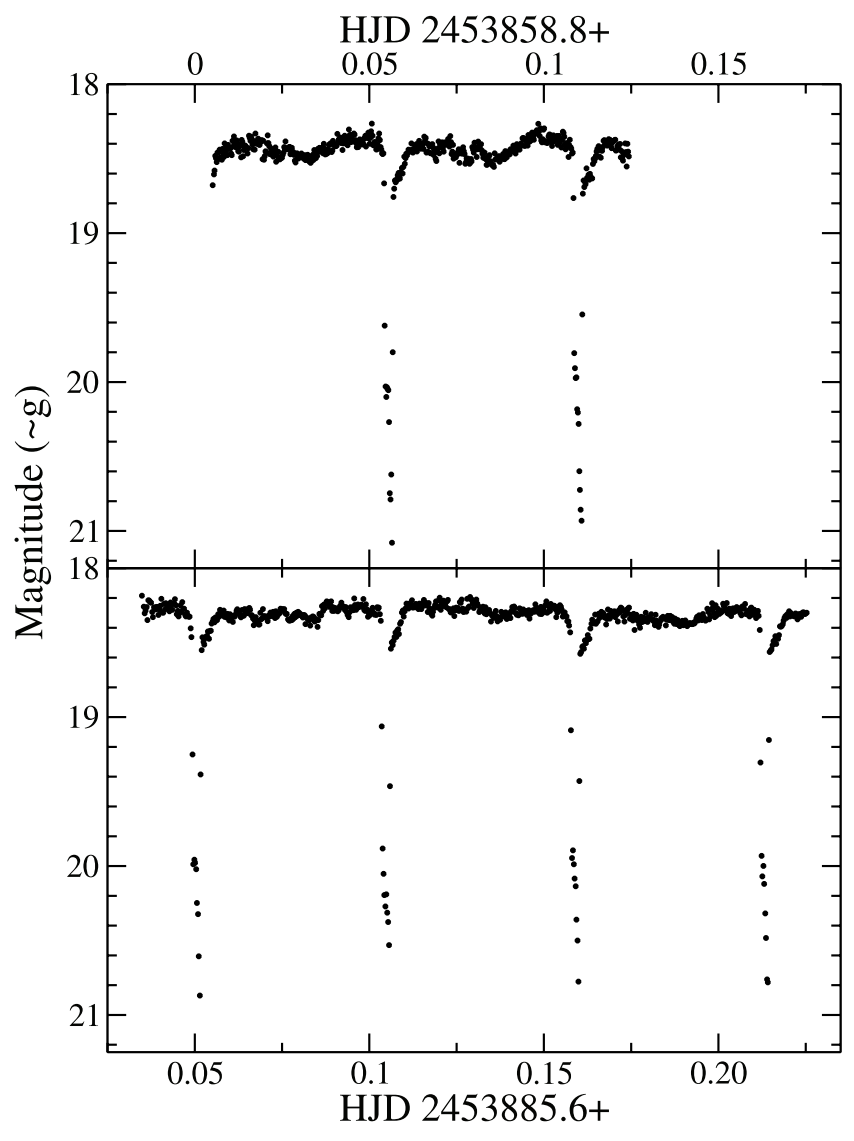

FIG. 3.-May 3 (top) and May 30 (bottom) light curves of SDSSJ 1433 showing the four observed eclipses. Error bars are $\leq 0.02 \mathrm{mag}$, except during eclipses when they are up to $0.2 \mathrm{mag}$.

to the opposite side of the disk. The May 3 light curve shows a more consistent hump feature than on May 30 , and there is evidence of a secondary eclipse feature as well. The eclipse itself is asymmetric and clearly shows a steep ingress due to the white dwarf eclipse, followed by a gradual ingress due to a spot and then a steep rise as the white dwarf reappears. Figure 4 shows the four eclipses from May 30 folded to match the ingresses and egresses. An almost linear decrease to the bottom of the eclipse is evident in the 3 minutes prior to the faintest magnitude. Higher time-resolution photometry in several colors will be able to constrain the geometry, as is done for other deeply eclipsing systems (Wood et al. 1989).

TABLE 5

SDSSJ 1433 ECLIPSE TIMES

\begin{tabular}{cc}
\hline \hline Eclipses (UT) & Eclipses (TCB) \\
\hline $08: 25: 17 \ldots \ldots \ldots \ldots \ldots . \ldots$ & $2,453,858.85686$ \\
\hline $09: 43: 25 \ldots \ldots \ldots \ldots .$. & $2,453,858.91112$ \\
\hline $03: 31: 19 \ldots \ldots \ldots \ldots .$. & $2,453,885.65180$ \\
\hline $04: 49: 23 \ldots \ldots \ldots \ldots .$. & $2,453,885.70602$ \\
\hline $06: 07: 31 \ldots \ldots \ldots \ldots . .$. & $2,453,885.76028$ \\
\hline $07: 25: 41 \ldots \ldots \ldots \ldots . .$. & $2,453,885.81455$ \\
\hline
\end{tabular}

Note.-Eclipse timings are mid-exposure deepest points of eclipse in UTC and Coordinated Barycentric Time.

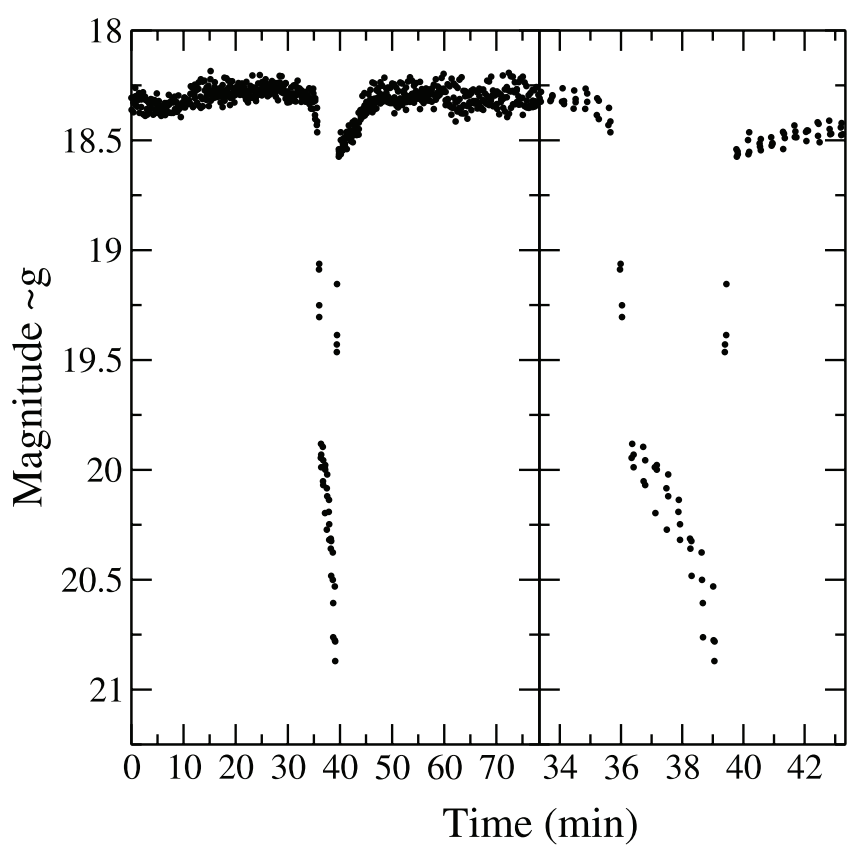

FIG. 4.-Four eclipses of SDSSJ 1433 on May 30 folded on the orbital period. The right panel is expanded around the eclipse to show the asymmetric structure during the faintest 3 minutes.

SDSSJ 1435.-The time-resolved spectra of SDSSJ 1435 show a large-amplitude sine wave that starts a repetition during the observation length of 108 minutes, with blue and red peaks clearly shifting back and forth during the interval. The best-fit periods for the $\mathrm{H} \alpha$ and $\mathrm{H} \beta$ lines are 83 and 74 minutes, so our estimate is $78 \pm 8$ minutes; longer data strings will be needed to refine this value further. Figure 5 shows the radial velocity curves.

\subsection{Dwarf Novae}

To identify a dwarf nova, several observations must be done in order to detect an outburst. The objects that are potential candidates are those that show large differences in their brightness between the SDSS photometry and spectroscopy. The Digitized Sky Survey (DSS) ${ }^{12}$ and $\mathrm{USNO}^{13}$ catalogs provide additional epochs to look for large brightness changes. One candidate dwarf

${ }^{12}$ Available at http://archive.stsci.edu/dss/.

${ }^{13}$ See http://www.nofs.navy.mil/projects/pmm/.

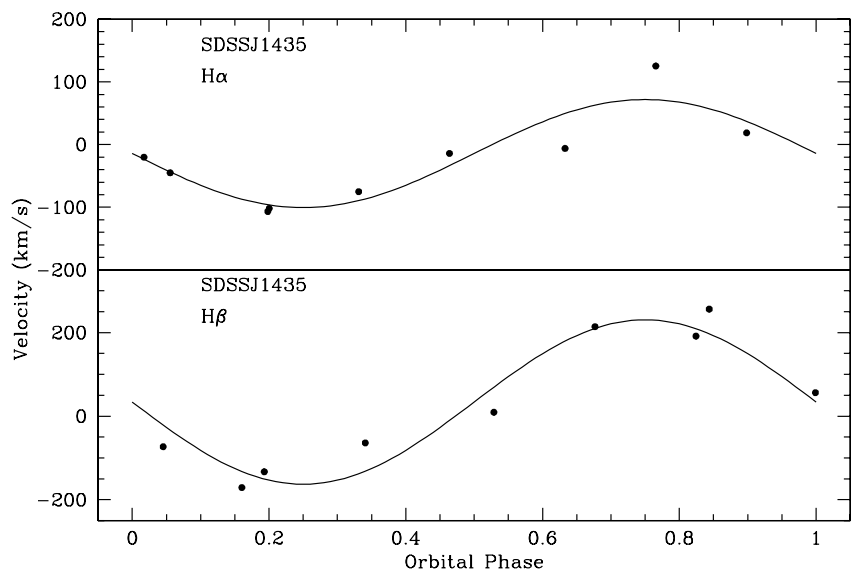

FIG. 5.- $-\mathrm{H} \alpha$ and $\mathrm{H} \beta$ velocity curves of SDSSJ 1435 with the best-fit sinusoids ( Table 4) superposed. 

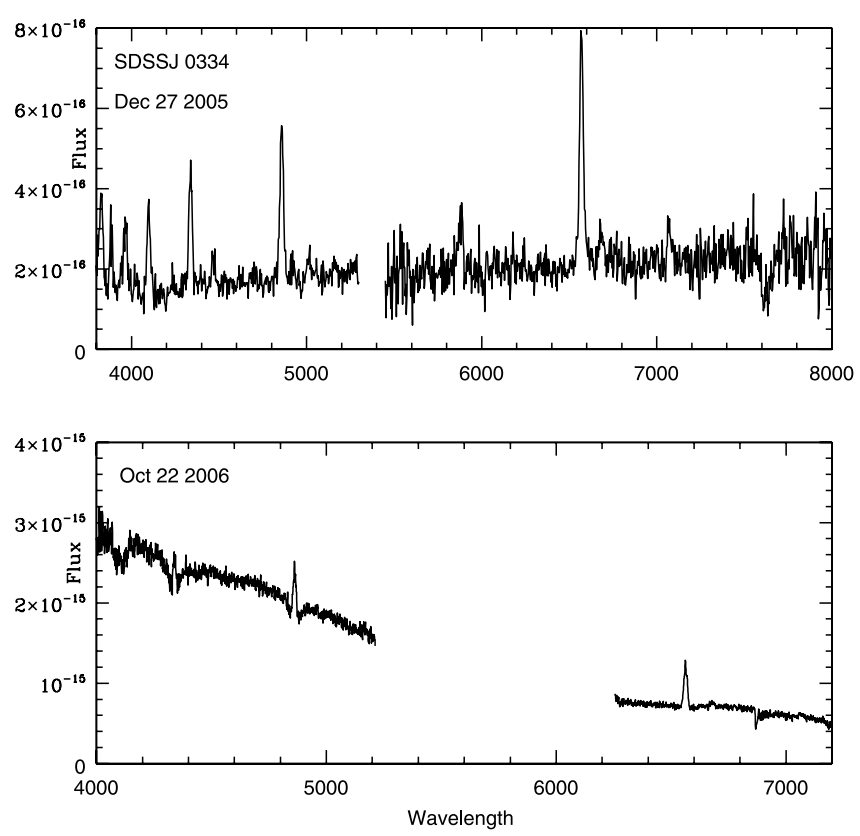

FIG. 6.-APO spectra of SDSSJ 0334 at quiescence on 2005 December 27 (top) and during an outburst on 2006 October 22 (bottom).

nova is SDSSJ 0837, whose SDSS spectrum (Fig. 1) is significantly fainter $(g \sim 20)$ than its SDSS $g$-magnitude of 19.0. Another good candidate is SDSSJ 0334, which we found during follow-up observations of bright UV excess sources and confirmed as a dwarf nova.

SDSSJ 0334. - The SDSS photometry of SDSSJ 0334 shows a bright object $(g=14.6)$, while spectroscopy at APO on 2005 December 27 shows an $\sim 18$ th magnitude source with the typical Balmer emission-line spectrum of a dwarf nova at quiescence (Fig. 6, top). This strong variability is consistent with the USNO catalog entries of $B=22.05$ and $R=17.8$. Additional photometry at NOFS on 2006 January 27 also showed the object at $V \sim 18$ (Fig. 7). APO time-resolved data obtained on 2006 October 22 (Fig. 6, bottom) showed an intermediate state $(V \sim 16)$, with the higher Balmer lines surrounded by absorption (likely from an extended accretion disk during a decline from outburst). The SRO photometry on 2006 February 3 and 4 also caught a decline from outburst, as the February 3 data showed a steady decline from $V \sim 16.26$ to 16.48 and the February 4 data continued the decline from $V \sim 17.4$ to 18.0 Thus, with twothirds of the observations obtained at quiescence and one-third at outbursts, it is likely that SDSSJ 0334 is a dwarf nova with a relatively frequent outburst timescale. The time-resolved spectra indicate an orbital period of 114 minutes for both $\mathrm{H} \alpha$ and $\mathrm{H} \beta$,

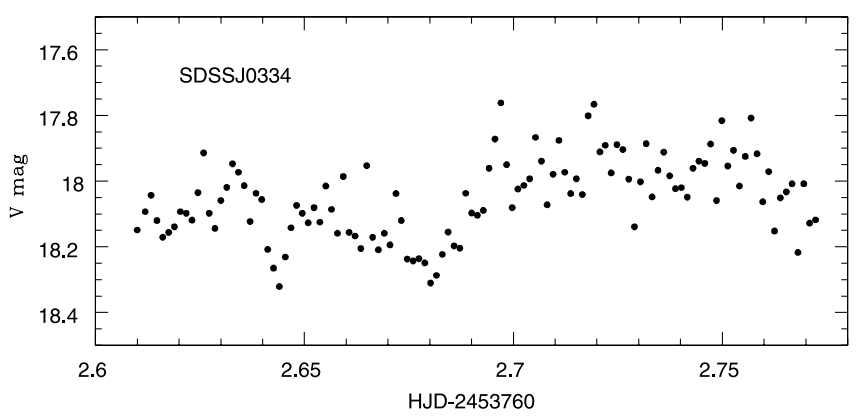

Fig. 7.-NOFS light curve of SDSSJ 0334 at quiescence on 2006 January 27. Error bars on each point are 0.03 mag.

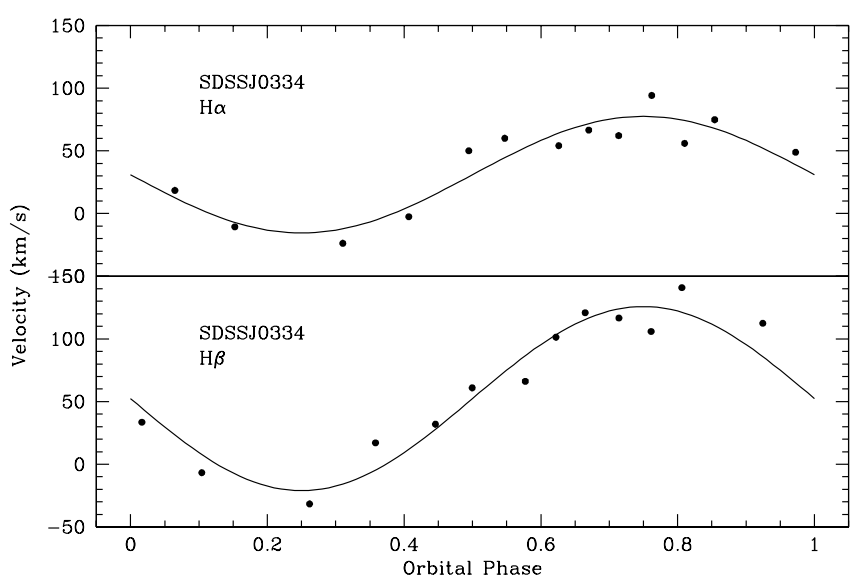

FIG. 8. - $\mathrm{H} \alpha$ and $\mathrm{H} \beta$ velocity curves of SDSSJ 0334 with the best-fit sinusoids (Table 4) superposed.

and the relatively low $K$ amplitude likely indicates a low-inclination system (Fig. 8 and Table 4). The quiescent light curve (Fig. 7) shows some low-amplitude ( $0.2 \mathrm{mag})$ modulation, but a longer time series is needed to see whether this repeats on the orbital timescale.

\subsection{Nova-likes with $\mathrm{He}$ II}

The known Polar SDSSJ 1554 shows a prominent highexcitation $\mathrm{He}$ II line compared to $\mathrm{H} \beta$ (Fig. 1) that is one of the identifying traits of Polars (Fig. 1). An even stronger ratio of $\mathrm{He}$ II $/ \mathrm{H} \beta$ is apparent in SDSSJ 0328, for which follow-up observations (see below) confirm a Polar nature as well. A weak He II emission line is the strongest line evident in SDSSJ 2243, but its spectrum (Fig. 1) shows a strong blue continuum with

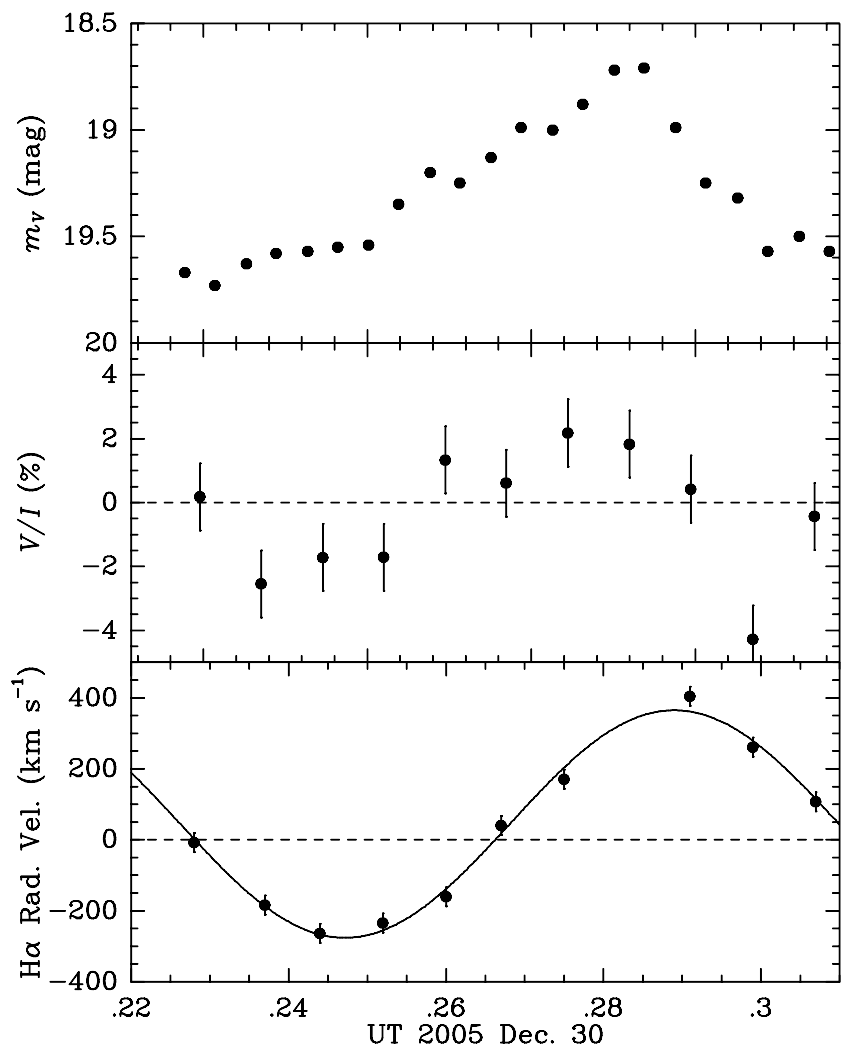

Fig. 9. $-V$ light, circular polarization, and $\mathrm{H} \alpha$ radial velocity curves of SDSSJ 0328 


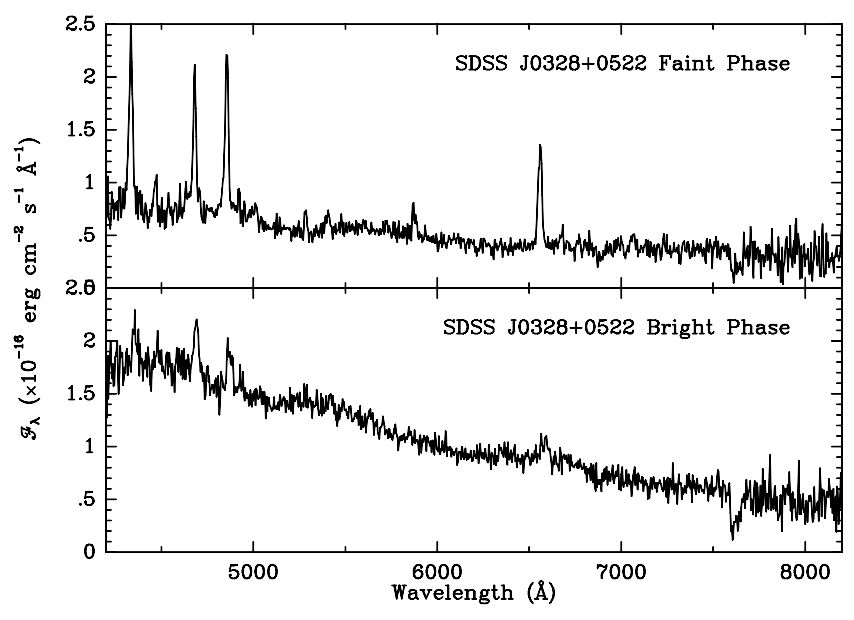

FIG. 10.-Bright- and faint-phase spectra of the Polar SDSSJ 0328 showing the changing cyclotron humps and strengths of the emission lines.

very weak Balmer lines. It is likely that SDSSJ 2243 is a novalike variable with an accretion disk and a high mass transfer rate. Although 10 spectra were obtained over a time span of almost $2 \mathrm{hr}$ (Table 2), the weakness of the lines and poor S/N prevented any determination of an orbital period. Two nights of SRO photometry showed only random variations at the $0.1 \mathrm{mag}$ level.

SDSSJ 0328.- Spectropolarimetric observations with the Bok telescope showed SDSSJ 0328 to be a polarized source that was clearly variable on a timescale of about $2 \mathrm{hr}$. The circular polarization summed over time and the entire spectrum has a value of $+0.48 \%$ and is larger at shorter wavelengths. Over the $2 \mathrm{hr}$ period, the polarization varies with an amplitude of $2.5 \%$. Figure 9 shows the visual magnitude and polarization change over the orbit. There is a bright phase that is about $1 \mathrm{mag}$ brighter than the rest of the orbit and lasts for about 0.55 of the orbit. This phase has positive circular polarization. Since the faint phase has negative polarization, it is clear that there are two actively accreting magnetic poles in this system. The spectrum shows clear cy-
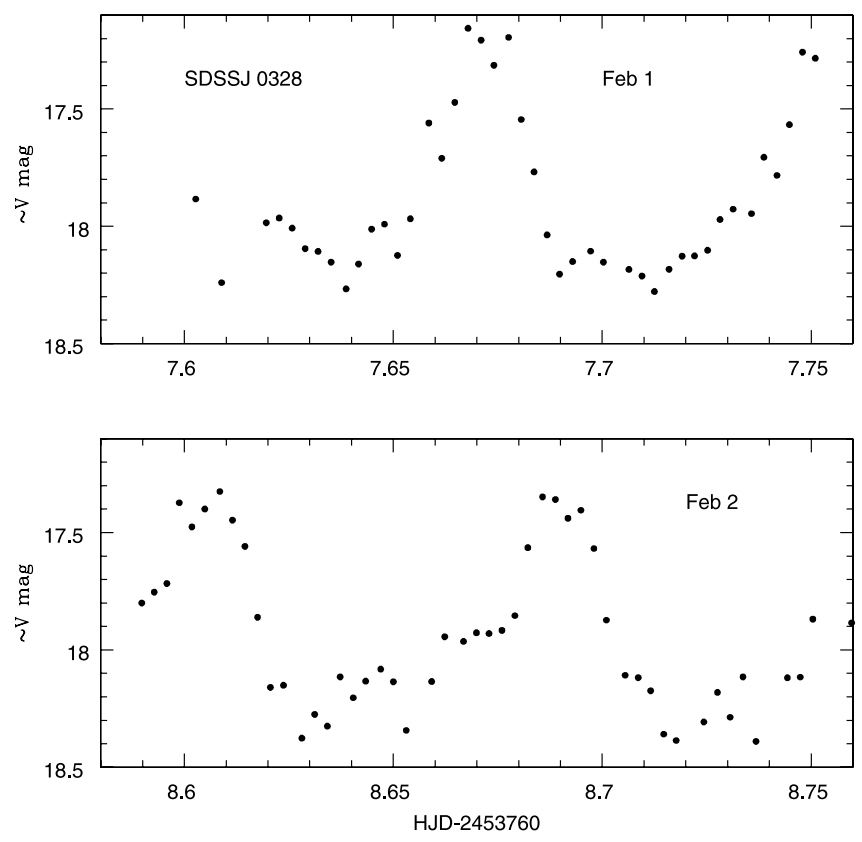

FIG. 11.-SRO photometry of SDSSJ 0328 showing the 1 mag variation throughout several orbits. Error bars are 0.04 at the peak of the modulation and up to $0.1 \mathrm{mag}$ at the minimum brightness.

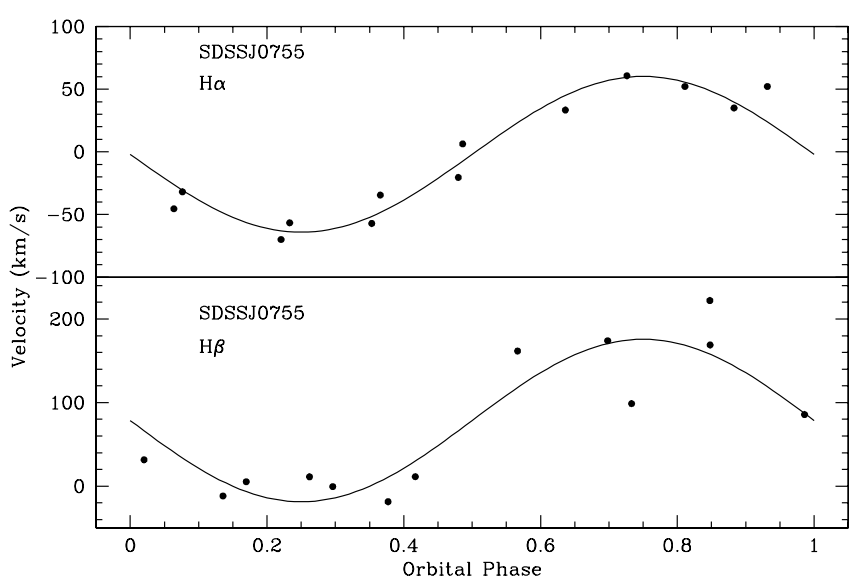

Fig. 12.- $\mathrm{H} \alpha$ and $\mathrm{H} \beta$ velocity curves of SDSSJ 0755 with the best-fit sinusoids ( Table 4) superposed.

clotron humps that move with phase. These harmonics are even visible in the survey spectrum (Fig. 1). Figure 10 shows spectra from the Bok telescope at the bright and faint phases. The faintphase spectrum shows cyclotron harmonics near 4750, 5600, and $6700 \AA$, which indicate a magnetic field of $33 \pm 5 \mathrm{MG}$. The line emission also changes dramatically throughout the orbit, almost completely disappearing at the peak of the bright phase, suggesting that the accretion funnel may be eclipsed by the secondary (or by the upstream funnel). However, since the cyclotron humps and circular polarization are still present, the white dwarf and accretion spot are not eclipsed.

A fit to the velocities measured from the $\mathrm{H} \alpha$ emission line produced the radial velocity curve shown in Figure 9. This provided a period of $120 \pm 9$ minutes and a semiamplitude of $320 \pm$ $55 \mathrm{~km} \mathrm{~s}^{-1}$. The systemic velocity crossing occurs very nearly at the middle of the bright phase. The timing implies that the accretion primarily feeds the bright pole. Two nights of SRO photometry (Fig. 11) a month later covered several orbits, and the large orbital modulation enabled a refinement of the period to $121.97 \pm 0.25$ minutes.

\subsection{Systems Showing the Underlying Stars}

If the disk does not dominate the total system light, the underlying white dwarf can be seen. This typically occurs for situations when the inclination is high (so that the edge rather than the face of the disk is the source) and/or the accretion rate is very low so that the disk is not optically thick and extensive. From

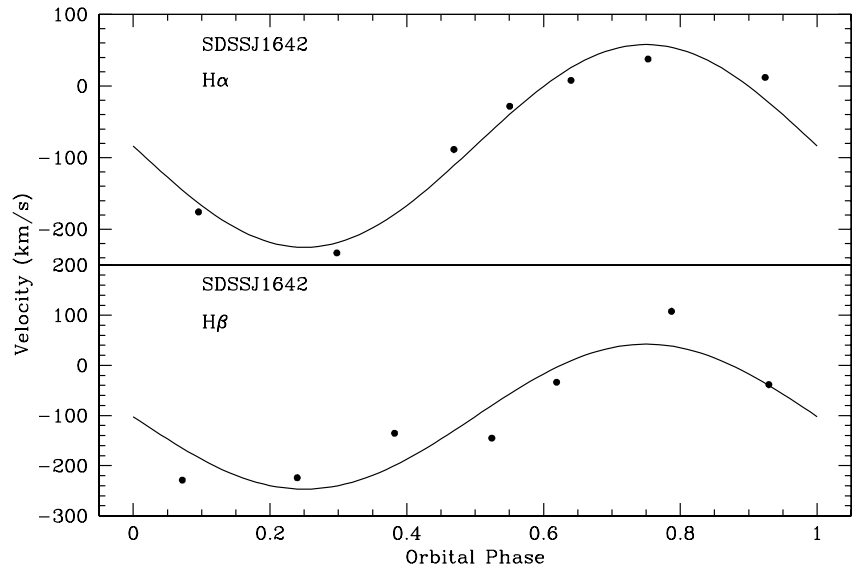

FIG. 13.- $\mathrm{H} \alpha$ and $\mathrm{H} \beta$ velocity curves of SDSSJ 1642 with the best-fit sinusoids ( Table 4) superposed. 
TABLE 6

ROSAT DETECTIONS

\begin{tabular}{ccccc}
\hline \hline RDSSJ & $\begin{array}{c}\text { ROSAT } \\
\left(\text { counts s }^{-1}\right)\end{array}$ & $\begin{array}{c}\text { Exp. } \\
(\mathrm{s})\end{array}$ & \multicolumn{1}{c}{ RXS } & Type \\
\hline $0945 \ldots \ldots \ldots$. & $0.03 \pm 0.01$ & 445 & $\mathrm{~J} 094558.3+292249$ & $\ldots$ \\
$1019 \ldots \ldots \ldots$. & $0.03 \pm 0.01$ & 415 & $\mathrm{~J} 101946.7+335811$ & $\mathrm{DN}$ \\
$1054 \ldots \ldots \ldots$. & $0.04 \pm 0.01$ & 369 & $\mathrm{~J} 105430.9+300620=$ SX LMi & DN \\
$1554 \ldots \ldots \ldots$. & $0.11 \pm 0.02$ & 381 & $\mathrm{~J} 155412.7+272143$ & Polar \\
\hline
\end{tabular}

${ }^{\text {a }}$ For a $2 \mathrm{keV}$ bremsstrahlung spectrum, 1 count $\mathrm{s}^{-1}$ corresponds to a $0.1-$ $2.4 \mathrm{keV}$ flux of about $7 \times 10^{-12} \mathrm{ergs}^{-2} \mathrm{~s}^{-1}$.

Figure 1 it is apparent that SDSSJ 0536, 0755, 1433, 1435, and 1559 show broad absorption lines that are likely from a white dwarf. Of these, SDSSJ 1433 and 1435 have been discussed as high-inclination systems. SDSSJ 0536 and 1559 show narrow emission, as well as absorption, indicative of irradiation of the secondary star by the white dwarf. Follow-up observations were obtained for SDSSJ 0755.

SDSSJ 0755. - We obtained 156 minutes of time-resolved APO spectroscopy of SDSSJ 0755; the data reveal a clear sinusoidal modulation of the $\mathrm{H} \alpha$ and $\mathrm{H} \beta$ emission lines that repeats during the observation interval. There are also narrow peaks within the emission lines that shift from blue to red on a similar timescale. The best-fit sine waves to the data (Fig. 12 and Table 4) give a period of 83 minutes for $\mathrm{H} \alpha$ and 87 minutes for $\mathrm{H} \beta$. While this difference can be a measure of our uncertainty, the $\mathrm{H} \alpha$ line is stronger and less contaminated by the underlying absorption and so may be a better indicator of the true period.

Figure 1 also reveals the presence of $M$ stars in the spectra of SDSSJ 1006 and 1554. As previously mentioned, SDSSJ 1554 is a known Polar, so there is no accretion disk to contribute light, although cyclotron emission is present. Thorstensen \& Fenton (2002) identified an M4 dwarf that produces the visible TiO bands. The TiO bands in SDSSJ 1006 indicate a spectral type of M2 ( \pm 1 spectral subclass) for its secondary.

The spectra of SDSSJ 0615 and 0805 show a downturn to the blue and absorption features indicative of an early $\mathrm{K}$ star. These systems could have long orbital periods or a secondary that evolved from a more massive progenitor (Thorstensen et al. 2002).

\subsection{Other Disk Systems}

The spectrum of SDSSJ 1107 has the appearance of a typical accretion disk system, with strong, broad Balmer emission lines. However, our 100 minutes of follow-up spectra showed no consistent radial velocity variation throughout this interval (both centroid and double-Gaussian methods of velocity determination were tried). The lines are clearly doubled and show narrow peak components during some spectra, which do not repeat a cycle during our observations, indicating that the period may be longer than most of the SDSS systems. It is also possible that the inclination is low so that the $K$ amplitude is small and the orbital velocity amplitude falls within our noise.

SDSSJ 1610 was first identified as KUV 16081+2229 and classified as an sdB (Wegner \& Swanson 1990). The width of the absorption lines makes a nova-like classification more reasonable. However, the weak lines will make determination of an orbital period very difficult.

SDSSJ 1642.-Figure 1 shows SDSSJ 1642 to be a typical disk system with a blue continuum and strong emission lines. Although the APO spectral coverage is short, both $\mathrm{H} \alpha$ and $\mathrm{H} \beta$ show a clear modulation from blue to red. Figure 13 shows the best-fit sine wave to the seven observed points. The period is close to the length of the data set (70 \pm 7 minutes from Table 4$)$, so a longer time series will be needed to further constrain the orbital parameters.

\subsection{ROSAT Correlations}

Four systems from Table 1 have X-ray detections $(>2.5 \sigma)$ within the positional errors of the ROSATAll Sky Survey (RASS; Voges et al. 1999, 2000). The X-ray count rates and exposure times for these sources are listed in Table 6 . The known Polar RX $\mathrm{J} 1554.2+2721$ is apparent, as are three other disk CVs. The new Polar SDSSJ 0328 was not detected, but it could have been in a low accretion state during the RASS.

\section{CONCLUSIONS}

The sixth year of SDSS operations has brought the total number of CVs in the SDSS database to 213, of which 177 are new discoveries. There are now $100 \mathrm{CVs}$ with known or estimated orbital periods. Of these, 70 are under $2 \mathrm{hr}, 10$ are in the period gap (mostly Polars), and 20 are long-period systems above the period gap. These numbers are now reaching sufficient statistical significance to be used in population studies. However, it should be kept in mind that SDSS is not complete in area; i.e., the selection of spectral fibers is based on a complicated mix of colors of quasars, white dwarfs, serendipity, and binary pairs. The final numbers will come from variability studies with followup spectra to determine the correct percentage of "missed" objects. Since the color range covered is large, the total numbers will change, but the mix of percentages is not expected to change by large amounts.

Among this data set are several objects of interest for future studies. The deep eclipse of SDSSJ 1433 could be parameterized with higher time resolution and multicolor photometry. A light curve for SDSSJ 1152 could reveal eclipses. The short-period systems showing the white dwarfs are prime candidates to search for pulsations of the white dwarf, as has been found in eight of the SDSS-discovered CVs (Warner \& Woudt 2004; Woudt \& Warner 2004; Gänsicke et al. 2006; Nilsson et al. 2006; Mukadam et al. 2007). The systems showing K stars (SDSSJ 0615 and 0805 ) should have high priority to determine whether their periods are under $2 \mathrm{hr}$ (indicating an abnormal evolution scenario) or several hours, as is typical for CVs with secondaries earlier than $\mathrm{M}$.

Funding for the SDSS and SDSS-II has been provided by the Alfred P. Sloan Foundation, the Participating Institutions, the National Science Foundation, the US Department of Energy, the National Aeronautics and Space Administration, the Japanese Monbukagakusho, the Max Planck Society, and the Higher Education Funding Council for England. The SDSS Web site is http://www.sdss.org.

The SDSS is managed by the Astrophysical Research Consortium for the Participating Institutions. The Participating Institutions are the American Museum of Natural History, the Astrophysical Institute Potsdam, the University of Basel, the University of Cambridge, Case Western Reserve University, the University of Chicago, Drexel University, Fermilab, the Institute for Advanced Study, the Japan Participation Group, Johns Hopkins University, the Joint Institute for Nuclear Astrophysics, the Kavli Institute for Particle Astrophysics and Cosmology, the Korean Scientist Group, the Chinese Academy of Sciences, Los Alamos National Laboratory, the Max Planck Institute for Astronomy, the Max Planck Institute for Astrophysics, New 
Mexico State University, Ohio State University, the University of Pittsburgh, the University of Portsmouth, Princeton University, the United States Naval Observatory, and the University of Washington.

We are grateful to John Thorstensen for useful comments on the manuscript. P. S. and A. M. acknowledge support from NSF grant AST 06-07840 and from NASA through Hubble Fellow- ship grant HST-HF-01175.01-A awarded by the Space Telescope Science Institute, which is operated by the Association of Universities for Research in Astronomy, Inc., for NASA, under contract NAS 5-26555. Studies of magnetic stars and stellar systems at Steward Observatory are supported by the NSF through AST 03-06080. M. O. was supported in part by Department of Energy contract DE-AC02-76SF00515.
Abazajian, K., et al. 2003, AJ, 126, 2081

2004, AJ, 128, 502

2005, AJ, 129, 1755

Adelman-McCarthy, J. K., et al. 2006, ApJS, 162, 38 2007, ApJS, in press

Aungwerojwit, A., et al. 2006, A\&A, 455, 659 (A06)

Fukugita, M., Ichikawa, T., Gunn, J. E., Doi, M., Shimasaku, K., \& Schneider, D. P. 1996, AJ, 111, 1748

Gänsicke, B. T., et al. 2004, ApJ, 613, L141 2006, MNRAS, 365, 969

Gunn, J. E., et al. 1998, AJ, 116, 3040 2006, AJ, 131, 2332

Hogg, D. W., Finkbeiner, D. P., Schlegel, D. J., \& Gunn, J. E. 2001, AJ, 122, 2129

Howell, S. B., Nelson, L. A., \& Rappaport, S. 2001, ApJ, 550, 897

Ivezic, Z., et al. 2004, Astron. Nachr., 325, 583

Jiang, X. J., Engels, D., Wei, J. Y., Tesch, F., \& Hu, J. Y. 2000, A\&A, 362, 263

Kato, T., \& Uemura, M. 2001, Inf. Bull. Variable Stars, 5097

Lupton, R. H., Gunn, J. E., Ivezic, Z., Knapp, G. R., \& Kent, S. M. 2001, in ASP Conf. Ser. 238, Astronomical Data Analysis Software and Systems X, ed. F. R. Harnden, Jr., F. A. Primini, \& H. E. Payne (San Francisco: ASP), 269

Lupton, R. H., Gunn, J. E., \& Szalay, A. 1999, AJ, 118, 1406

Mukadam, A., et al. 2007, ApJ, submitted

Nilsson, R., Uthas, H., Ytre-Eide, M., Solheim, J.-E., \& Warner, B. 2006, MNRAS, 370, L56

Pier, J. R., Munn, J. A., Hindsley, R. B., Hennessy, G. S., Kent, S. M., Lupton, R. H., \& Ivezic, Z. 2003, AJ, 125, 1559

Richards, G. T., et al. 2002, AJ, 123, 2945

Schmidt, G. D., Szkody, P., Henden, A., Anderson, S. F., Lamb, D. Q., Margon, B., \& Schneider, D. P. 2007, ApJ, 654, 521

\section{REFERENCES}

Schmidt, G. D., et al. 2005, ApJ, 630, 1037

Schwope, A. D., Schreiber, M. R., \& Szkody, P. 2006, A\&A, 452, 955

Shafter, A. W. 1983, ApJ, 267, 222

Smith, J. A., et al. 2002, AJ, 123, 485

Stoughton, C., et al. 2002, AJ, 123, 485

Szkody, P., et al. 2002, AJ, 123, 430

- 2003a, ApJ, 583, 902

2003b, AJ, 126, 1499

2004, AJ, 128, 1882

2005, AJ, 129, 2386

2006, AJ, 131, 973

Thorstensen, J., \& Fenton, W. H. 2002, PASP, 114, 74

Thorstensen, J., Fenton, W. H., Patterson, J. O., Kemp, J., Krajci, T., \& Baraffe, I. 2002, ApJ, 567, L49

Tovmassian, G. H., Greiner, J., Zharikov, S. V., Echevarria, J., \& Kniazev, A. 2001, A\&A, 380, 504

Tucker, D., et al. 2006, Astron. Nachr., 327, 821

Voges, W., et al. 1999, A\&A, 349, 389 2000, IAU Circ. 7432

Wagner, R. M., et al. 1998, AJ, 115, 787

Warner, B. 1995, Cataclysmic Variable Stars (Cambridge: Cambridge Univ. Press)

Warner, B., \& Woudt, P. 2004, in ASP Conf. Ser. 310, Variable Stars in the Local Group, ed. D. W. Kurtz \& K. R. Pollard (San Francisco: ASP), 382 Wegner, G., \& Swanson, S. R. 1990, AJ, 100, 1274

Wickramasinghe, D. T., \& Ferrario, L. 2000, PASP, 112, 873

Wood, J. H., Horne, K., Berriman, G., \& Wade, R. A. 1989, ApJ, 341, 974

Woudt, P., \& Warner, B. 2004, MNRAS, 348, 599

York, D. G., et al. 2000, AJ, 120, 1579 